\title{
Der militärische Einsatz bewaffneter Drohnen
}

\author{
Zwischen Schutz für Soldaten und gezieltem Töten
}

Bernhard Koch, Institut für Theologie und Frieden, Herrengraben 4, 20459 Hamburg (koch@ithf.de)

Bernhard Rinke, Zentrum für Demokratie- und Friedensforschung, Universität Osnabrück (brinke@uni-osnabrueck.de)

Mit dem Einsatz bewaffneter Drohnen hat eine neue Qualität der Kriegsführung begonnen. Vor diesem Hintergrund untersucht der Beitrag mit Fokus auf die deutsche Debatte, ob und inwiefern der militärische Einsatz bewaffneter Drohnen als legitim gerechtfertigt werden kann. Genauer wird der militärische Einsatz bewaffneter Drohnen aus ethischer Perspektive im Spannungsfeld zwischen dem Schutz für Soldaten einerseits und gezieltem Töten andererseits analysiert. Wir argumentieren, dass der Einsatz bewaffneter Drohnen bedenklich ist, da der Schutz für die eigenen Soldatinnen und Soldaten durch den Einsatz dieser Technologie verbessert werden mag, aber mit einer erhöhten Gefahr für Leib und Leben feindlicher Soldatinnen und Soldaten einhergeht.

\section{The military use of armed unmanned aerial vehicles}

Between protection of soldiers and targeted killing

The use of armed unmanned aerial vehicles (UAVS) introduced a new era of robotic warfare. Against this background, this article examines whether and to what extent the military use of armed UAVs can be legitimated, focusing on the German debate. More precisely, the military use of armed UAVs is analyzed from an ethical point of view in the field of tension between protecting soldiers on the one hand and targeted killing on the other hand. We argue that the use of armed UAVS is alarming, because the protection of a country's own soldiers might be improved by using this technology, however, at an increased danger for life and limb of the enemy's soldiers.

Keywords: armed unmanned aerial vehicles, targeted killing, robotic warfare, ethics

\section{Einleitung}

Für den ehemaligen Bundesminister der Verteidigung, Thomas de Maizière, waren bewaffnete Drohnen in einem ersten Statement als solche ,ethisch neutral“ (Jungholt und Meyer 2012).

This is an article distributed under the terms of the Creative Commons Attribution License CCBY 4.0 (https://creativecommons.org/licenses/by/4.0/)

https://doi.org/10.14512/tatup.27.3.38

Submitted: 30.07.2018. Peer reviewed. Accepted: 08.10.2018
Hinter diesem Urteil verbirgt sich der Gedanke, es könnte eine Art ethische Unbedenklichkeitserklärung für ein technisches Instrument ausgestellt werden (Grunwald 2013, S. 6), das gleichermaßen für gute und schlechte Zwecke, in guten und schlechten Handlungen eingesetzt werden kann. In der Tat beurteilt angewandte Ethik zunächst einmal Handlungen und nicht äußere Gegenstände wie etwa Drohnen. Wenn ein Handlungstyp als solcher ethisch erlaubt oder unerlaubt ist, dann scheint er es unabhängig von den konkreten technischen Mitteln, mit denen er ausgeführt wird, zu sein - außer die Mittel verändern den Handlungstyp selbst. Wir wollen mit unserem Beitrag an dieser Stelle ansetzen; unsere Überlegungen verfolgen das Anliegen, den militärischen Einsatz bewaffneter Drohnen im Spannungsfeld zwischen Schutz für Soldaten und gezieltem Töten ethisch zu analysieren. Reine Aufklärungs- bzw. Beobachtungsdrohnen sind nicht Gegenstand dieses Beitrags, auch wenn die Aufklärungsfähigkeit von Drohnen militärisch sogar bedeutsamer als die Zuschlagsfähigkeit sein mag (so jedenfalls Scharre 2018, S. 14).

Um es vorweg zu nehmen: Zum einen wird in diesem Beitrag die These vertreten, dass es irreführend ist, davon zu sprechen, bewaffnete Drohnen seien als solche ,ethisch neutral“. Zum anderen wird das Argument entfaltet, dass derjenige ethisch zu kurz greift, der zugunsten dieses militärischen Instruments nur vorbringt, es ließe sich mit ihrem Einsatz der Schutz von Soldatinnen und Soldaten verbessern. Vielmehr vertreten wir die Auffassung, dass der Erwerb und der Einsatz bewaffneter militärischer Drohnen sehr kritisch betrachtet werden muss.

Unser Beitrag gliedert sich wie folgt: Im ersten Schritt werden wir kurz Handlungen als Gegenstand der ethischen Reflexion genauer betrachten. Im zweiten Schritt werden wir das Argument vortragen, dass Drohnen keineswegs ethisch neutral, sondern vielmehr sogar ein bevorzugenswertes technisches Instrument der Kriegführung seien. Zur Entkräftung dieser Argumentation werden wir im dritten Schritt zunächst den Begriff des Schutzes problematisieren und in einem vierten Schritt dann die Problematik gezielter Tötungen in den Blick nehmen. Auf diese Weise wird auch deutlich, dass das Verständnis von Technikethik, das hier eingebracht werden soll, nicht einfachhin eines 
ist, das aus deduktiven Schlussfolgerungen apodiktische normative Lösungen erreichen kann, sondern dass vielmehr in einem hermeneutischen Durchgang bereits vorgebrachte Argumente auf ihren begrifflichen Gehalt und ihre normativen Voraussetzungen befragt werden. Dies ermöglicht eine ethisch informierte politische Entscheidung über Erwerb und Einsatz von bewaffneten Drohnen, aber es erzwingt sie nicht.

\section{Handlungen als Gegenstand der ethischen Reflexion}

In der ethischen Beurteilung von technischen Instrumenten ist man zunächst mit folgendem Problem konfrontiert: Primärer Gegenstand ethischer Reflexion ist die normative Bewertung menschlicher Handlungen, nicht von Dingen, Werkzeugen, technischen Instrumenten oder gar Waffen. Dennoch beurteilen wir auch Werkzeuge oder sogar hochtechnologische Instrumente als „,moralisch gut" oder ,ethisch schlecht“. Wenn wir etwa eine Waffe als ethisch verwerflich einstufen, dann wohl vorrangig deshalb, weil sie Handlungen ermöglicht, die wir als ethisch verwerflich qualifizieren. Wir gestehen aber zu, dass sich auch mit einer Waffe (jedenfalls in einzelnen Fällen) ethisch gut handeln lässt. Die Beurteilung der Handlung ist so besehen folglich in der Lehre vom Gerechten Krieg ihre klassische Form angenommen hat (Walzer 2015; Janssen und Quante 2017) - mit Blick auf die Anwendung militärischer Gewalt im Krieg die Einhaltung des Diskriminierungsgebotes eingefordert, demzufolge zwischen Kombattanten und Nicht-Kombattanten unterschieden werden muss. Mehr noch: Gefordert wird sogar das Verbot von Waffen, die das Diskriminierungsgebot nicht einhalten können (Haspel 2002, S. 133 ff.). Art. 35,2 des 1. Zusatzprotokolls zu den Genfer Konventionen (GK 1977) verbietet sogar jeden Einsatz von Waffen, ,die geeignet sind, überflüssige Verletzungen oder unnötige Leiden zu verursachen“. Art. 36 schreibt vor, dass jede neue Waffentechnologie daraufhin überprüft werden muss, ob mit ihr das Humanitäre Völkerrecht eingehalten werden kann.

Nun gibt es aber kaum ein technisches Instrument, welches grundsätzlich und immer nur in schlechten Handlungen genutzt werden könnte. Solch ein Instrument wäre dann wohl wirklich malum in se. Meistens lassen sich sogar Waffen für gute und schlechte Handlungszwecke einsetzen. Trotz der insofern möglicherweise günstigen Wirkungen, die man mit Waffen erreichen kann, bedeutet dies aber nicht - so unsere These -, dass Waffen ,ethisch neutral“ seien. Zwar könnte man mit einer Pistole vielleicht auch Walnüsse knacken, aber wohl niemand würde eine Pistole ausschließlich zu diesem Zweck erwerben. Es ge-

\section{Wenn wir also bestimmte technische Erfindungen als ethisch verwerflich qualifizieren, dann deshalb, weil mit ihnen überwiegend schlecht gehandelt wird.}

auch technikethisch vorrangig. Dieser Beitrag fokussiert mithin auf die Betrachtung ethischer Fragen ,für Menschen im Umgang mit Maschinen“ (Misselhorn 2018, S. 8; kursiv im Original), also die Frage, wie ,die Menschen mit einer bestimmten Technologie“ - in unserem Fall die Drohnentechnologie - ,aus moralischer Sicht umgehen sollten“ (ebd., S. 8). Insofern versteht sich der vorliegende Aufsatz auch nicht als Beitrag zur Maschinenethik, deren „Gegenstand die Entwicklung einer Ethik für Maschinen ist" (Misselhorn 2018, S. 8, Hervorhebung im Original; zur Maschinenethik vgl. auch Wallach und Allen 2009 sowie Bendel 2018) und somit im Grunde dem Feld der Medienethik zuzuordnen ist (Rath et al. 2018).

Wenn wir also bestimmte technische Erfindungen - wie z. B. Landminen - als ethisch verwerflich qualifizieren, dann deshalb, weil mit ihnen überwiegend schlecht gehandelt wird. So gelten Landminen als ,,inhumane“ Waffentechnologie, weil mit ihnen unterschiedslos (indiskriminierend) Kombattanten (,Soldaten“), Nicht-Kombattanten (z.B. Militärärzte und -geistliche) und Zivilpersonen in und nach einem bewaffneten Konflikt getötet werden bzw. getötet werden können (Schörnig 2013, S. 49). Jedenfalls wird in der friedensethischen Gewaltkritik - welche hört schon zur Natur des Waffeneinsatzes, dass mit ihm Gewalt ausgeübt wird, und Gewalthandlungen sind zweifellos in besonderer Weise ethisch rechenschaftsbedürftig. Jedoch können gewisse Gewalthandlungen auch gerechtfertigt werden, z. B. als Notwehr oder Nothilfe.

\section{Bewaffnete militärische Drohnen als ethisch bevorzugenswertes technisches Instrument der Kriegsführung}

Wenn wir nun angesichts der oben formulierten Überlegungen die Frage beantworten wollen, ob bewaffnete militärische Drohnen ethisch neutral sein können, müssen wir uns zunächst vergegenwärtigen, dass ,Militärtechnik (...) ein breiter Bereich verschiedenartiger Anwendungen (ist), die durch die Aufgaben der Streitkräfte bestimmt werden“ (Altmann 2013, S. 324). Für unseren Zusammenhang ist dabei von besonderer Bedeutung, dass sich der Einsatz bewaffneter Drohnen im Spannungsfeld von Schutz- und Tötungshandeln vollzieht. Jedenfalls wird häufig argumentiert, ,bewaffnete Drohnen dienten in ganz beson- 
derer Weise dem Schutz der eigenen Soldatinnen und Soldaten“ (Schörnig 2013, S. 47). So hat etwa Generalleutnant Karl Müllner (von April 2012 bis Mai 2018 Inspekteur der Luftwaffe) die Auffassung vertreten, der Einsatz bewaffneter Drohnen bzw. „(f)erngesteuerte(r) Luftfahrzeuge mit Bewaffnungsoption“ sei „militärisch notwendig“, um die eigenen Soldatinnen und Soldaten (besser) schützen zu können (Müllner 2015). Müllner nimmt den moralischen Standpunkt dessen ein, der Verantwortung für jene trägt, die ihm unterstellt sind. In der Tat liegt im Schutz der eigenen Soldatinnen und Soldaten ein ethischer Wert. Und wer würde bestreiten, dass militärische Robotik z. B. bei der Minenräumung Menschen schützen kann. Aber bewaffnete Drohnen einzusetzen, ist nicht nur Minenräumung.

Wenn man einen militärischen Auftrag hat, geht es darum, diesen mit Mitteln auszuführen, die einen selbst, diejenigen, für die man Verantwortung trägt und Unbeteiligte an der Auseinandersetzung - die Zivilisten - bestmöglich vor den potenziellen Folgen der Anwendung von Gewalt bewahren bzw. schützen. Aber auch die Gegner sind zu berücksichtigen; unnötige Gewalt ist auch im Krieg nicht zulässig.

Wenn diese instrumentelle Sichtweise so zutreffen würde, würde also mit einer weiteren „Automatisierung in der Militär- und Waffentechnik (...) die Gefahr, der Soldaten ausgesetzt sind“, zunächst einmal reduziert (Franke 2016, S. 29). Denn, so Sauer und Schörnig (2012, S. 363): „Machines can operate in hazardous environments“, mit der Folge, dass , ,[...] the use of unmanned systems in dangerous situations such as forward reconnaissance, bomb disposal, or the suppression of enemy air defense means that human soldiers can be given the best possible force protection" (ebd., S. 364). Schutzhandlungen gelten jedoch gemeinhin als ethisch erlaubt oder gar geboten, da das Militär auf diesem Wege der „Fürsorgepflicht" nachkommt, die es „für sein Personal“ besitzt (Misselhorn 2018, S. 178). Entsprechend hat etwa der Moralphilosoph Bradley Strawser denn auch argumentiert, dass die moralische Pflicht zum Einsatz bewaffneter Drohnen bestehe: ,„...) I argue that we have a duty to protect an agent engaged in a justified act from harm to the greatest extent possible, so long as the protection does not interfere with the agent's ability to act justly" (Strawser 2010, S. 342). Mit Blick auf dieses Argument hat Niklas Schörnig schließlich zudem festgestellt: „Selbst Kritiker müssen anerkennen, dass dieser Schutzgedanke in westlichen Demokratien relevant ist, die ihre Soldatinnen und Soldaten als Bürger in Uniform und nicht als Kanonenfutter verstehen" (Schörnig 2013, S. 47).

Doch nicht nur im Blick auf den Schutz der eigenen Soldaten scheinen Kampfdrohnen ein unter ethischen Gesichtspunkten bevorzugenswertes Instrument der Kriegsführung zu sein: So wird weiterhin das Argument ins Feld geführt, dass die Beschaffung von Kampfdrohnen „ethisch geboten“ sei, weil durch sie unbeabsichtigte Schäden (,Kollateralschäden“) geringer gehalten werden können als mit anderen Waffen. Sie werden in ihrer Präzision sogar als „ein exaktes Gegenstück zu den ethisch in besonderem Maße verwerflichen Massenvernichtungsmitteln angesehen" (Alwardt 2013, S. 4).
Wenn wir diese Befunde zusammennehmen, handelt es sich bei bewaffneten Drohnen also keineswegs um ein neutrales ethisches Instrument. Sie wären augenscheinlich ein zu bevorzugendes Instrument der Kriegsführung. Zumindest kann man sich mit durchaus bedenkenswerten Gründen für die Nutzung bewaffneter Drohnen einsetzen. Allerdings würde es die Reflexion arg verkürzen, wenn man glaubt, an dieser Stelle bereits ein Ergebnis in der Hand zu haben. Denn bisher wurde eben nur ein Ausschnitt aus dem Bild dargestellt, das man in den Blick nehmen muss, wenn man zu einem abgewogenen ethischen Urteil kommen will. Insbesondere ist mehr als zweifelhaft, ob man die meisten Einsätze bewaffneter Drohnen überhaupt als ,schützende Handlungen“ bezeichnen kann.

\section{Der problematische Begriff des Schutzes}

In diesem Sinne ist zunächst zu klären, was „Schutz“ im hier betrachteten Kontext überhaupt bedeutet. Denn bewaffnete Drohnen sind ja kein Schild, das zwischen einem möglichen Opfer und einem Aggressor eine Sperre aufbauen würde, sondern mit bewaffneten Drohnen werden Angreifer und Gegner aktiv bekämpft. Dies kann unter Umständen ethisch legitim sein, aber dafür muss zuerst die Frage beantwortet werden, unter welchen Umständen es überhaupt legitim ist, Gewaltmittel gegen andere Personen einzusetzen. Diese Debatte kann hier nicht in ihren Einzelheiten nachgezeichnet werden (zum jüngeren Diskussionsstand Frowe 2016 und Rudolf 2017). Grundsätzlich gilt verteidigende Gewalt dann als erlaubt, wenn sie legitime Rechtsgüter - insbesondere Leib und Leben - zu bewahren versucht.

Dessen unbenommen muss jedoch auch das Schutzbedürfnis auf seine Legitimität hin befragt werden, denn nicht jedem Schutzbedürfnis darf einfachhin nachgegeben werden. Nur weil ich mich von meinem stets etwas zornig wirkenden Nachbarn bedroht fühle, darf ich ihn noch lange nicht angreifen - weder mit einer Drohne noch mit anderen Mitteln. Anders gewendet: Nur, weil in westlichen Demokratien das Schutzbedürfnis hoch ist, heißt das nicht, dass man um jeden Preis schützen darf.

Die zentrale Schwierigkeit liegt nun darin, dass "Schutz" oder „Sicherheit“" Begriffe sind, die in ethischen Debatten kaum echtes kritisches Potenzial entfalten können. Zu unbestimmt ist ihr jeweiliger Gehalt, und zu leicht werden sie verwendet, um bereits vorhandene Voreinstellungen zu begründen.

Natürlich lässt sich einerseits argumentieren, dass sich die Gewaltanwender durch den Einsatz bewaffneter Drohnen der Gegengewalt entziehen können und dadurch besser geschützt sind. Es mag auch stimmen, dass man durch bewaffnete Drohnen näher an das Ziel der Gewaltanwendung herankommen kann und dadurch die Zahl kollateraler Opfer sinkt, was man unter Umständen als verbesserten „Schutz“ verstehen mag, obwohl es sich in erster Linie natürlich um Angriffshandlungen handelt. Andererseits jedoch kann dem entgegengehalten werden, dass die Drohnenkriegsführung in der Konsequenz zur Folge hat, 
dass Staatsgrenzen leichter überflogen werden können, sich dadurch Zonen bewaffneter Konflikte ausgedehnt haben und somit der Schutz, den das Kriegsvölkerrecht, also das Humanitäre Völkerrecht, vormals gewährt hat, nun verwässert worden ist (Schaller 2011; zur völkerrechtlichen Debatte insgesamt Frau 2014).

Hinzu kommt schließlich, dass die Unschärfe des Begriffes „Schutz“ einhergeht mit der Unschärfe des Begriffes „Bedrohung“, der eng oder weit gefasst werden kann (vgl. Rudolf 2017, S. 118 f.). Zumindest stellt sich die Frage, ob „Schutz“ nur im Falle einer unmittelbaren, direkten tödlichen Bedrohung legitim ist, oder ob das Streben nach „Schutz“ auch dann Legitimität beanspruchen kann, wenn die Bedrohung eher diffus und ihre Eintrittswahrscheinlichkeit schwierig zu kalkulieren ist.

Die Begriffe „Schutz“ oder „Sicherheit“" haben eine Tendenz, ethisch zu „totalisieren“. Es lässt sich immer noch weiter schützen oder immer noch ein Quantum an Sicherheit zulegen. In der Tat darf ein demokratischer Rechtsstaat seine Bürger nicht schutzlos lassen. Das gilt nicht zuletzt für die Bürgerinnen und Bürger, die in seinen Diensten stehen und angesichts ihrer Aufgaben mitunter Gefahrensituationen ausgesetzt sind, in denen besonderer Schutzbedarf besteht: Polizistinnen und Polizisten und eben Soldatinnen und Soldaten. Ganz zweifellos haben Soldatinnen und Soldaten einen Anspruch auf möglichst gute Ausrüstung. Dennoch muss über dieses „,möglichst gut“ in einer Abwägung mit anderen Gütern, für die der Staat ebenfalls Sorge trägt, entschieden werden. Nicht jeder Ministerialbeamte kann einen gepanzerten Dienstwagen erhalten, obwohl es natürlich sehr beklagenswert ist, wenn ein Ministerialbeamter in einem schwächer geschützten Fahrzeug verletzt wird oder ums Leben kommt, was mit einem besser geschützten Fahrzeug verhindert worden wäre. Aber der Staat muss auch die Kosten der Dienst- ist Ausdruck einer solchen technischen Fähigkeit. Erst recht ist es natürlich das Erbauen von Waffensystemen, mit denen man aus der Distanz operieren kann. Nur zeigt sich eben, dass sich die Verwundbarkeit trotz des technischen Instruments nur verlagert, sie aber nicht verschwindet. Ferngesteuerte Systeme sind immer anfällig für Ausfälle bei der Funkverbindung. Solche Ausfälle oder Störungen können sogar mutwillig von Gegnern herbeigeführt werden: das sogenannte jamming. Manchmal wird ein Szenario skizziert, bei dem ferngesteuerte Systeme gewissermaßen von außen ,übernommen“ werden, indem man eben die Verbindung „,kapert“ (Alwardt 2013, S. 4; Scharre 2018, S. 13 u. 15). Die Waffe, die man selbst auf seine Gegner richten wollte, wird nun von eben diesem Gegner gegen einen selbst gewendet.

\section{Gezielte Tötungen}

Die westliche Öffentlichkeit hat von den bewaffneten Drohnen als militärischem Instrument vor allem in Beziehung auf die von der Obama-Administration verfolgte Praxis ,gezielter Tötungen“ - ,verstanden als die im Auftrag von Staaten durchgeführte geplante Tötung bestimmter einzelner Personen, die nicht inhaftiert sind“" (Rudolf 2017, S. 115 f.) in Afghanistan und Pakistan Kenntnis genommen. Diese Eindrücke haben das Bild der bewaffneten Drohne nachhaltig geprägt. In Deutschland bemühen sich Militär und Sicherheitspolitik vor diesem Hintergrund darauf hinzuweisen, dass bewaffnete Drohnen in der Bundeswehr nicht in diesem Sinne eingesetzt werden sollen. Beispielsweise hielt Generalleutnant Karl Müllner ausdrücklich fest: „Für uns geht es dabei nicht um die rechtlich und ethisch problema-

\section{Die Drohnenkriegsführung hat zur Folge, dass sich Zonen bewaffneter Konflikte ausdehnen und der Schutz, den das Humanitäre Völkerrecht gewährt, verwässert ist.}

wagenflotte gegen das Schutzbedürfnis seiner Bediensteten abwägen dürfen. In diesem Sinne ist der Staat nicht verpflichtet, jedes technische Instrument, das den Schutz seiner Soldaten oder Polizisten verbessern könnte, auch tatsächlich anzuschaffen. Aber freilich geraten der Staat und seine Entscheidungsträger in Rechtfertigungszwänge, wenn es zu einem Schaden an Leib und Leben kommt. Häufig kann aber das Handeln, das vom Versuch, umfassenden Schutz zu erhalten, Abstand genommen hat, gerechtfertigt werden (vgl. Koch 2015).

Menschen suchen Schutz und Sicherheit, weil sie von Natur aus verwundbar sind. Aber dieser Verwundbarkeit werden eben nun die technischen Fähigkeiten des Menschen entgegengestellt. Auch die Rechtskonstruktion, z. B. das Schaffen des Humanitären Völkerrechts, aber auch des innerstaatlichen Polizeirechts, tische Einsatzpraxis anderer Staaten - Stichwort targeted killing - sondern ausschließlich darum, die vom Deutschen Bundestag erteilten Einsatzaufträge möglichst effektiv und mit dem geringsten verantwortbaren Risiko für unsere Soldaten sowie für die unbeteiligte Zivilbevölkerung erfüllen zu können“ (Müllner 2015).

Es ist dennoch unumgänglich, gezielte Tötungen bei den Drohnen mit zu reflektieren (vgl. zur moralischen und rechtlichen Problematik gezielter Tötungen exemplarisch die jüngere Diskussion bei Rudolf 2017, S. 114 ff. sowie Schockenhoff 2018, S. 705 ff.), denn die Handlungstypen gehen in einem militärischen Einsatz ineinander über. Hier haben wir es mit einem „Slippery Slope“-Argument („Dammbruchargument“, siehe Walton 1992) zu tun: Die technische Dynamik treibt einen Pro- 
zess voran, der zu einem anfänglich abgelehnten Zustand führt. Solche „Slippery-Slope“-Argumente sind in der Technikethik sehr häufig und sie schließen nur, wenn der Endzustand tatsächlich ethisch unakzeptabel ist.

Hier soll also keineswegs bestritten werden, dass Drohnen in einem gewissen Sinn schützen, so wie auch Luftnahunterstützung ,schützt“. Aber es ist ein zu verkürztes Bild, nur auf diesen Schutz zu schauen und nicht zu sehen, welche Folgen Drohneneinsätze an anderer Stelle haben: Wenn beispielsweise die Folge von Drohneneinsätzen darin liegt, dass mehr Personen auf der Seite des Gegners zu angreifbaren Zielen erklärt werden, dann ist der Schutz auf der einen Seite (der eigenen) mit geringerem Schutz auf der anderen Seite (der des Gegners) ,erkauft“. Ethik
Nun sind gezielte Tötungen nach dem Recht der bewaffneten Konflikte, also dem Humanitären Völkerrecht, nicht immer illegal. Sie wären unerlaubt, wenn sie geschützte Personen beträfen, aber gegen gegnerische Kombattanten oder Zivilisten, die aufgrund ihrer direkten Beteiligung an den Feindseligkeiten ihren Schutz verloren haben, sind sie rechtlich legal. Voraussetzung ist natürlich immer, dass die Gewaltintensität die Schwelle zum bewaffneten Konflikt überschritten hat und das Humanitäre Völkerrecht daher überhaupt Anwendung finden kann. Aber diese Schwelle ist nirgendwo klar definiert und auch nicht definierbar. Dadurch aber entstehen Interpretationsräume, die Akteure gerne zu ihren Gunsten nutzen. Ist nämlich die Schwelle zum bewaffneten Konflikt nicht überschritten, müssen menschenrecht-

\section{Durch die Verfügbarkeit eines technischen Instruments kann sich der normative Kontext schleichend ändern.}

muss aber alle Seiten in den Blick nehmen. Bewaffnete Drohnen erlauben die Nachverfolgung von entdeckten Gegnern auch über weite Strecken und über Landesgrenzen hinaus. Irgendwo auf diesem Weg geht das gewissermaßen „herkömmliche“ Töten eines Kriegsgegners (das man übrigens ethisch auch problematisieren muss) in ein ,gezieltes Töten“ über.

Nils Melzer, der die wegweisende völkerrechtliche Arbeit zu dieser Praxis verfasst hat, definiert diese anhand von fünf Definitionsmerkmalen: 1. Der Gebrauch von tödlicher Gewalt, 2. das Ziel des Tötens, 3. das Anvisieren individuell ausgewählter Personen, 4. der Umstand, dass die angezielten Personen nicht in Gewahrsam des Angreifers sind, und 5. der Umstand, dass die Handlung des gezielten Tötens einem Völkerrechtssubjekt zugeschrieben werden kann (vgl. Melzer 2008, S. 3 f.). Es ist zu beachten, dass es sich nur um Definitions- und nicht um Legitimationskriterien handelt und unter den Definitionsmerkmalen kein Bezug auf bestimmte technische Instrumente genommen wird. Gezieltes Töten kann mit bewaffneten Drohnen durchgeführt werden, muss aber nicht. Bei der Tötung von Osama bin Laden am 2. Mai 2011 handelte es sich gewiss um ein ,gezieltes Töten“ in den Definitionsgrenzen, die Melzer aufstellt, aber es erfolgte durch Schusswaffen in der Hand einer Spezialeinheit der US-Navy Seals. Es zeigt sich jedoch, dass bewaffnete Drohnen einfach technologisch für ein gezieltes Töten bestens geeignet sind. Mit ihnen kann ein gutes Lagebild entwickelt werden, und mit ihnen können einzelne Personen halbwegs ordentlich erkannt werden. Nach wie vor gibt es Fehler, aber es ist zu erwarten, dass sich die Erkennungstechnologie noch weiter verbessert. Der Zusammenhang von gezielten Tötungen und bewaffneten Drohnen ist also nur begrifflich kontingent, empirisch ist er nicht zufällig. Denn „die militärische Logik erfordert letztlich die volle Ausnutzung der Fähigkeiten eines Systems“ (Koch und Schörnig 2015, S. 2), und zur vollen Nutzung von bewaffneten Drohnen gehört das gezielte Töten dazu. liche Standards zur Anwendung kommen, und das heißt, dass die Gefangennahme der Tötung immer vorgezogen werden muss. Gefangennehmen kann aber eine bewaffnete Drohne, die über Westpakistan kreist und von den USA aus gesteuert wird, nicht Daher ist es auch rechtlich fast so gut wie ausgeschlossen, dass bewaffnete Drohnen in einem polizeilichen Einsatz genutzt werden. Einzig unmittelbare Bedrohungssituationen, die einen finalen Rettungsschuss erlauben, könnten dafür in Frage kommen.

Um den gezielten Tötungen rechtliche Legitimation zu verschaffen, ist es daher nötig, die Bedingungen für einen bewaffneten Konflikt möglichst niedrig zu hängen. Es scheint auch in der Tat einiges darauf hinzudeuten, dass man heute leichter bereit ist, auch bei geringeren militärischen Auseinandersetzungen von einem bewaffneten Konflikt zu sprechen als man es vor 20 oder 30 Jahren getan hätte. Wir sehen also, dass sich durch die Verfügbarkeit eines technischen Instruments der normative Kontext schleichend ändern kann. Schon in diesem Sinne sind bewaffnete Drohnen also nicht ,ethisch neutral“. Allerdings stellt sich noch ein größeres Problem: Die rechtliche Legalität garantiert noch keine ethische Legitimität. So ist zwar das Recht nicht unerheblich für die Moral, aber Rechtskonformität für sich ist noch nicht ethisch ausreichend. Möglicherweise erlaubt das $\mathrm{Hu}-$ manitäre Völkerrecht zu viel.

In der Tat lässt sich argumentieren, dass die in Melzers Definitionskriterien genannte zweite Bedingung für das ,gezielte Töten“ ethisch höchst problematisch ist. Dass man handelt mit dem Ziel des Tötens - anders gesagt: dass die Handlung nur dann ,,erfolgreich“ ist, wenn die angegriffene Person tot ist -, das kommt nur im Falle des Vollzugs der Todesstrafe vor. In allen anderen Fällen der Anwendung letaler Gewalt ist das Töten zwar häufig wahrscheinlich und in Kauf genommen, aber nicht das Ziel des Handelns selbst. Einen erfolgreichen finalen Rettungsschuss gibt man auch dann ab, wenn die Bedrohung beseitigt ist, der ursprüngliche Bedroher aber überlebt. Das Humanitäre Völkerrecht 
(St.Petersburger Erklärung von 1868) hat nur das Kampfunfähigmachen als Ziel militärischer Handlungen ausgegeben, nicht die Tötung selbst. Drohneneinsätze laufen aber zuweilen so ab, dass man dann, wenn man bemerkt hat, dass die Gegner nicht getötet worden sind, noch einmal in einem zweiten Schlag (double tape) nachsetzt. Zu solchen „Doppelschlägen“ sind kaum öffentliche Zahlen verfügbar. Allerdings hat die Nichtregierungsorganisation The Bureau of Investigative Journalism (2013) versucht, einige Fälle dieser Art zu dokumentieren. Solche Strafaktionen (von „Verteidigung“ ist hier kaum mehr zu sprechen) geschehen unilateral, ohne wirkliche Strafautorität und ohne der angegriffenen Person eine Chance zur Verteidigung in einem geordneten Strafverfahren zu geben. Diese Praxis ist ethisch höchst bedenklich, ja, im Grunde ausgeschlossen.

Wir wissen, dass man dem Anwender einer bestimmten Technologie nicht a priori unterstellen kann, dass er die Technologie zu illegitimen Zwecken einsetzt. Aber man darf einen Anwender schon darauf hinweisen, dass ein technisches Instrument bestimmte Anwendungen nahelegt, in die man geradezu nolens volens hineinrutschen kann. Denn so wenig die Unterstellung gerechtfertigt ist, so wenig ist es auch die überzogene Selbstsicherheit, man werde das Gerät schon korrekt verwenden, wenn es einmal zur Verfügung steht.

\section{Fazit und Ausblick:}

\section{Können bewaffnete militärische Drohnen ethisch neutral sein?}

Tragen wir die Befunde unserer Überlegungen abschließend noch einmal kurz zusammen:

Bei bewaffneten Drohnen handelt es sich weder um ein ethisch neutrales technisches Instrument noch handelt es sich aus ethischer Perspektive um ein nachgerade besonders zu bevorzugendes Instrument der Kriegsführung. Vielmehr sind bewaffnete Drohnen ethisch sehr bedenklich, da der Schutz, der auf der einen Seite für die eigenen Soldatinnen und Soldaten durch den Einsatz dieser Technologie verbessert werden mag, auf der anderen Seite mit einer Auflösung von Schutz einhergeht - insbesondere dadurch, dass das Humanitäre Völkerrecht etwas von seinem Biss verliert, weil seine Grundunterscheidungen wie die nach Zivilisten und Kombattanten oder die nach Kriegsgebiet und außerhalb des Kriegsgebiets liegende Zonen verwässert werden.

Nun bedeuten die hier von uns betrachteten ferngesteuerten Flugsysteme mit Bewaffnung nur einen Zwischenschritt auf dem Weg zu mehr Entscheidungsautonomie von Waffensystemen. Bewaffnete Drohnen können als eine Art „Einstiegsdroge“ in Richtung auf den Einsatz vollautomatisierter und autonomer Waffensysteme (killer robots) gesehen werden. Mit anderen Worten: Auch der Weg von der bewaffneten Drohne zum autonomen Waffensystem kann mit großer Plausibilität im Sinne einer, ,Slippery Slope“-Argumentation dargestellt werden, aber diese Diskussion würde den hier zur Verfügung stehenden Rahmen sprengen.

\section{Literatur}

Altmann, Jürgen (2013): Militärtechnik. In: Armin Grunwald (Hg.): Handbuch Technikethik. Stuttgart: Metzler, S. 324-329.

Alwardt, Christian et al. (2013): Braucht Deutschland Kampfdrohnen? Hamburger Informationen zur Friedens- und Sicherheitspolitik 50/2013. Online verfügbar unter https://ifsh.de/pdf/publikationen/hifs/HI_50_Kampfdrohnen.pdf, zuletzt geprüft am 09.07.2018.

Bendel, Oliver (2018): Überlegungen zur Disziplin der Maschinenethik. In: Aus Politik und Zeitgeschichte 68 (6-8), S. 34-38.

Franke, Ulrike (2016): Automatisierte und autonome Systeme in der Militär- und Waffentechnik. In: Aus Politik und Zeitgeschichte 66 (35-36), S. 28-32.

Frau, Robert (Hg.) (2014): Drohnen und das Recht. Völker- und verfassungsrechtliche Fragen automatisierter und autonomer Kriegsführung. Tübingen: Mohr Siebeck.

Frowe, Helen (2016): The ethics of war and peace. An Introduction. 2. ed. London: Routledge.

GK - Genfer Konventionen (1977): 1. Zusatzprotokoll. Online verfügbar unter https://www.admin.ch/opc/de/classified-compilation/19770112/index.html, zuletzt geprüft am 05.10.2018.

Grunwald, Armin (Hg.) (2013): Handbuch Technikethik. Stuttgart: Metzler. Haspel, Michael (2002): Friedensethik und Humanitäre Interventionen. Der Kosovo-Krieg als Herausforderung evangelischer Friedensethik. Neukirchen-Vlyn: Neukirchener Verlag. Janssen, Dieter; Quante, Michael (Hg.) (2017): Gerechter Krieg. Ideengeschichtliche, rechtsphilosophische und ethische Beiträge. 2. Auflage. Paderborn: mentis.

Jungholt, Thorsten; Meyer, Simone (2012): De Maizière wirbt für Einsatz bewaffneter Drohnen. In: Die Welt, veröffentlicht am 03. 08.2012. Online verfügbar unter https://www.welt.de/politik/deutschland/article108473948/ De-Maiziere-wirbt-fuer-Einsatz-bewaffneter-Drohnen.html, zuletzt geprüft am 05.10.2018.

Koch, Bernhard (2015): Bewaffnete Drohnen und andere militärische Robotik. Ethische Betrachtungen. In: Christof Gramm und Dieter Weingärtner (Hg.): Moderne Waffentechnologie. Hält das Recht Schritt? Baden-Baden: Nomos, S. 32-56.

Koch, Bernhard; Schörnig, Niklas (2015): Die Gefahren autonomer Waffensysteme. Online verfügbar unter http://www.juspax-eu.org/de-wAssets/docs/ Tree//Tree-Of-Justice/PEACE/Ethics-of-peace/JPE-MEMO-1-LAWS-DE.pdf, zuletzt geprüft am 28. 07.2018.

Melzer, Nils (2008): Targeted killing in international law. Oxford: Oxford University Press.

Misselhorn, Catrin (2018): Grundfragen der Maschinenethik. Stuttgart: Reclam.

Müllner, Karl (2015): Ferngesteuerte Luftfahrzeuge für unsere Soldaten im Einsatz. Veröffentlicht bei kreuz-und-quer.de. Diskussionsforum zum politischen Handeln aus christlicher Verantwortung. 26. Januar 2015. Online verfügbar unter https://blogkreuzundquer.files.wordpress.com/2014/12/ mc3bcllner-drohnen.pdf, zuletzt geprüft am 11. 07.2018.

Rath, Matthias; Karmasin, Matthias; Krotz, Friedrich (2018): Brauchen Maschinen Ethik? Begründungstheoretische und praktische Herausforderungen. In: Dies. (Hg.): Maschinenethik. Normative Grenzen autonomer Systeme. Wiesbaden: Springer VS, S. 1-10.

Rudolf, Peter (2017): Zur Legitimität militärischer Gewalt. Bonn: Bundeszentrale für politische Bildung. 
Sauer, Frank; Schörnig, Niklas (2012): Killer drones. The ,silver bullet' of democratic warfare? In: Security Dialogue 43 (4), S. 363-380.

Schaller, Christian (2011): Gezielte Tötungen und der Einsatz von Drohnen. Zum Rechtfertigungsansatz der Obama-Administration. In: Humanitäres Völkerrecht-Informationsschriften (HuV-I) 24, S. 91-96.

Scharre, Paul (2018): Army of none. Autonomous weapons and the future of war. New York: W. W. Norton.

Schockenhoff, Eberhard (2018): Kein Ende der Gewalt? Friedensethik für eine globalisierte Welt. Freiburg i. B.: Herder.

Schörnig, Niklas (2013): „Aber wehe, wehe, wehe! Wenn ich auf das Ende sehe!" Gefahren der Beschaffung bewaffneter Drohnen. In: Marc von Boemcken, Ines-Jacqueline Werkner, Magret Johannsen und Bruno Schoch: Friedensgutachten 2013. Berlin: Lit, S. 46-57.

Strawser, Bradley J. (2010): Moral predators: The duty to employ uninhabited aerial vehicles. In: Journal of Military Ethics 9 (4), S. 342-368.

The Bureau of Investigative Journalism (2013): Get the data. The return of double-tap drone strikes. Online verfügbar unter https://www.thebureauinvestigates.com/stories/2013-08-01/get-the-datathe-return-of-double-tap-drone-strikes, zuletzt geprüft am 05.10.2018.

Wallach, Wendell; Allen, Colin (2009): Moral machines. Teaching robots right from wrong. Oxford: Oxford University Press.

Walton, Douglas (1992): Slippery slope arguments. Oxford: Clarendon Press.

Walzer, Michael (2015): Just and unjust wars. A moral argument with historical

illustrations. 5. Auflage. New York: Basic Books.

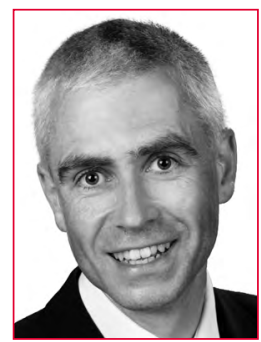

\section{DR. BERNHARD KOCH}

ist stellvertretender Leiter des Instituts für Theologie und Frieden in Hamburg sowie Lehrbeauftragter für Philosophie an der Universität Hamburg und der Goethe-Universität Frankfurt am Main.

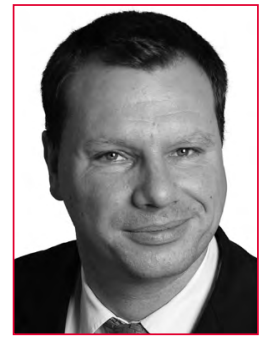

\section{DR.BERNHARD-WILHELM RINKE}

ist externer Projektmitarbeiter des Instituts für Theologie und Frieden in Hamburg, Mitglied des Zentrums für Demokratie- und Friedensforschung an der Universität Osnabrück sowie Lehrbeauftragter am dortigen Institut für Sozialwissenschaften. Aktuell beschäftigt er sich vor allem mit Fragen der ethisch legitimen Anwendung militärischer Gewalt. Unter dem Arbeitstitel „Ethische Fragestellungen im Kontext autonomer Waffensysteme" hat er gemeinsam mit Bernhard Koch vom Institut für Theologie und Frieden in Hamburg jüngst ein Gutachten für das Büro für Technikfolgen-Abschätzung beim Deutschen Bundestag verfasst.

\section{politische ökologie \\ Die Buchreihe für Querdenker und Vordenkerinnen}

\section{Smartopia}

\section{Geht Digitalisierung auch nachhaltig?}

Algorithmen überwachen und lenken zunehmend den Alltag. Wenige Großkonzerne machen gute Geschäfte mit unseren Daten, während sie vollmundig smarte technische Lösungen mit geringeren Ressourcenverbräuchen versprechen. Die Politik schaut beinahe hilflos zu, wie sich die Digitalisierung rasant und weitgehend unkontrolliert Bahn bricht. Dabei werden umweltschonende Lebensstile mithilfe digitaler Tools theoretisch einfacher. Praktisch besteht aber die Gefahr, guten Gewissens weiter zu viel zu konsumieren und damit noch mehr Energie und Rohstoffe zu verbrauchen, als der Planet auf Dauer verkraften kann.
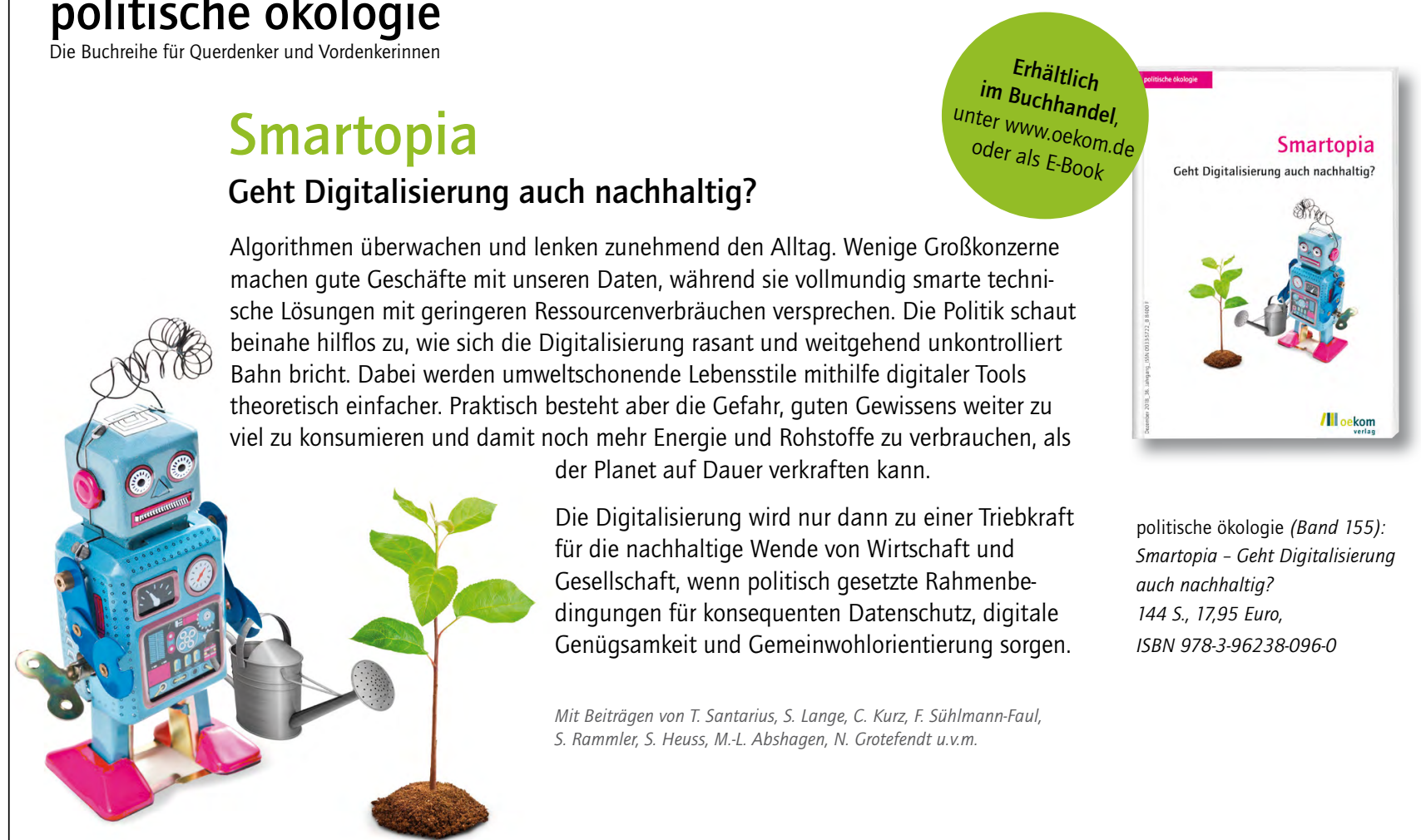
Die Digitalisierung wird nur dann zu einer Triebkraft für die nachhaltige Wende von Wirtschaft und Gesellschaft, wenn politisch gesetzte Rahmenbedingungen für konsequenten Datenschutz, digitale Genügsamkeit und Gemeinwohlorientierung sorgen.

politische ökologie (Band 155): Smartopia - Geht Digitalisierung auch nachhaltig? 144 S., 17,95 Euro, ISBN 978-3-96238-096-0 